\title{
PROPUESTA DE ESPECIFICADORES DIAGNÓSTICOS VINCULADOS AL ESTRÉS Y EL TRAUMA: UNA APORTACIÓN A LA NOSOLOGÍA PSICOPATOLÓGICA
}

\author{
ANTONIO TALARN, NURIa NAVARRo, LAURA RosSEll y ANNA RigaT \\ Universidad de Barcelona
}

\begin{abstract}
Resumen: En la actualidad poseemos numerosa evidencia empírica que demuestra que muchos cuadros psicopatológicos están influidos por el estrés y/o los traumas. Sin embargo, los manuales de diagnóstico de los sistemas DSM y CIE no siempre permiten establecer esta relación de un modo adecuado y preciso. A partir de una definición operativa de los conceptos de estrés y trauma se proponen una serie de especificadores que podrian ser incluidos en el eje I de estos sistemas diagnósticos. Los especificadores propuestos son: 1 ) especificadores del estrés: cuando un trastomo se vincula con estrés agudo, crónico, o a un trauma y 2) especificadores de factores asociados al estrés: como factor predisponente, desencadenante o de mantenimiento. El uso de estos especificadores mejoraria la precisión diagnóstica, permitirfa disponer de más datos sobre las relaciones entre estrés y psicopatología, y resultaría beneficioso para la terapéutica de los pacientes.
\end{abstract}

Palabras clave: Estrés, trauma, especificadores diagnósticos.

\section{A proposal of diagnostic specifiers related to stress and trouma: A contribution to psychopathological nosology}

\begin{abstract}
Considerable empirical evidence is now available to show that many psychopathological conditions are influenced by stress and/or traumas. However, the diagnostic manuals of the DSM and ICD systems do not always allow us to establish this relation in a precise and adequate way. Starting from working definitions of stress and trauma, we propose a series of diagnostic specifiers which can be included in the I axis of these diagnostic systems. The proposed specifiers are: 1) stress specifiers: when a disorder is linked to acute stress, chronic stress or trauma and 2) specifiers of factors associated with stress: predisposition, trigger factors or maintenance. The use of these specifiers would improve diagnostic accuracy, disclose more information regarding the relations between stress and psychopathology and would be beneficial in terms of patient therapy.
\end{abstract}

Keywords: stress, trauma, diagnostic specifiers.

\section{INTRODUCCIÓN}

Actualmente es una evidencia probada que el estrés, ya sea en forma de respuesta a sucesos vitales menores (DeLonguis, Lazarus y Folkman, 1988), mayores (Holmes y David, 1898), o a los traumas (Davidson y Foa, 1993; van der Kolk, McFarlane y Weisaeth, 1996), presenta una notable relación con los trastornos psicopatológicos (Barret, Rose y Klerman, 1979; Buendia, 1993; Jarne, 2000; Neufeld, 1982; Sandín y Chorot, 1993).

\section{Recibido 5 julio 2005; aceptado 10 marzo 2006.}

Correspondencia: Antonio Talarn, Dpto. de Personalidad, Facultad de Psicologia (Universidad de Barcelona), Avda. del Valle Hebron 171,08035 Barcelona.Corroo- : atalarn@ubedu
El presente trabajo parte de estas evidencias para poner de relieve que los manuales diagnósticos habituales sólo reconocen esta relación de modo muy fragmentario y para ciertos cuadros clínicos. Aunque en su descargo puede argumentarse que se trata de manuales de clasificación, sin referencias a la etiología de los trastornos que mencionan, una revisión más detallada muestra que esta perspectiva no siempre se mantiene. Por ello presentamos una definición operativa de los conceptos de «estrés» y utrauma» con la intención de efectuar una propuesta de especificadores diagnosticos vinculados a ambos conceptos. Mediante el uso de espocificadores de este tipo de problemas psicosociales sería posible sefialar con más precisión su presencia en numerosos cuadros psicopatológicos. 
Y de esta mayor precisión se podrían derivar notables beneficios tanto a nivel científico (en tanto se dispondría de más datos sobre estas relaciones) como a nivel terapéutico (ya que los pacientes podrían recibir una orientación terapéutica más acorde a sus condicionantes psicosociales).

\section{PLANTEAMIENTOS PREVIOS}

Si atendemos al concepto de estrés en términos mas generales observaremos que su relación con los trastornos mentales tiene una larga tradición en la historia de la psicopatología. Sin afán de efectuar una revisión exhaustiva podemos citar a Karl Jaspers (1913) y su concepto de «reacción»(conducta psicopatológica en respuesta a las condiciones ambientales) en cierto modo opuesto al concepto de «proceso» (situación mórbida como resultado de un proceso endógeno). En la misma línea de Jaspers, Kurt Schneider (1946) describió las «reacciones vivénciales anormales» y los «desarrollos vivénciales reactivos» basándose en las respuestas del sujeto a situaciones de estrés mas o menos crónicas. Recogiendo las ideas de los dos autores citados Adolf Meyer (1957) postuló que prácticamente toda la psicopatología era de origen reactivo, vinculada con episodios y situaciones de estrés (mediada a través de procesos psicobiológicos). Como es bien sabido Meyer ejerció una notable influencia en la psiquiatría norteamericana y sus ideas quedaron plasmadas la primera edición del DSM (APA, 1952).

Desde la psicología la situación es muy similar. Hace años que se insiste en el papel fundamental del estrés en tanto que factor patógeno de suma importancia para las condiciones psicopatológicas. Recogiendo las influencias seminales de autores como Pavlov (1927) («neurosis experimental») y Selye $(1936,1956)$ («síndrome general de adaptación»), la psicologia ha efectuado aportaciones muy sustanciales en este sentido. El detallaje y evaluación de los acontocimientos vitales (Holmes y Rahe, 1967) - las ideas de Lazarus (1966) sobre el estrés y los mecanismos de afrontamiento son solo dos éjemplos significativos de lo que estamos señalando.

Con respecto al estrés producido como consecuencia de la exposición a un trauma la historia se repite. Esto es, hay un sinfin de autores y numerosa evidencia empírica que sustentan la idea de que los traumas pueden ser una importante variable patogénica en muchas alteraciones psicopatológicas y no sólo en aquellas claramente identificadas como tales, es decir, el «trastorno por estrés agudo» y el "trastorno por estrés postraumático».

Al igual que en el caso del estrés en términos generales, al producido por los acontecimientos traumáticos no le falta tradición en la psicopatologia. Kraepelin (1883) mencionó la uneurosis de espanto"; Oppenheimer en 1892, (citado por McFarlane, 2004) acuñó el termino «neurosis traumática», otorgándole entidad de trastorno molecular del sistema nervioso, debido al shock recibido. Este diagnóstico fue reconocido por Kraepelin en la 60 edición de su «Tratado de Psiquiatria» (Kraepelin, 1899). Incluso desde el psicoanálisis más temprano, a pesar de la conocida renuncia de Freud (1897) a la «teoría de la seducción" se insistió en que el trauma debía de ser considerado en la etiología de los trastornos mentales y del comportamiento (Ferenczi, 1908; 1932). En plena segunda guerra mundial Kardiner (1941) escribio The traumatic neurosis of war donde describió los síntomas propios del estrés postraumático incluida la amnesia.

En la actualidad disponemos de datos suficientes como para afirmar que la presencia de traumas es de importancia, tal como decíamos, no solo en lo que podríamos denominar «trastomos postraumáticos» («trastorno por estrés agudo» y « trastorno por estrés postraumático «) sino en muchos otros. Así, por ejemplo, Beck y Van der Kolk (1987) encontraron que el $46 \%$ de las mujeres psicóticas crónicas hospitalizadas tenían historial de incesto infantil. Muenzenmaier, Meyer y Struening (1993) relatan que el 65\% de los pacientes entrevistados durante un año en un ambulatorio habían sufrido abusos durante la infancia. Friedman (1996) señala que hasta el $80 \%$ de los pacientes diagnosticados con «trastorno por estrés postraumáticon presentan comorbilidad con, al menos, otro trastorno mental, y sugiere que la enorme posibilidad de error diagnóstico que se puede dar con estos pacientes impide la adecuada apreciación del factor traumático en la génesis de sus trastornos. Mueser, Salyers y Rosenberg (2001) hallaron antecedentes traumáticos en el $90 \%$ de los pacientes de un programa de asistencia a drogadictos con trastornos mentales. Tucker (2002), por ejemplo, señala que hay muchos pacientes en los que no se efectúa un diagnostico correcto debido a que se obvian los factores traumáticos y estresantes en sus historiales e interrogatorios. En nuestro entorno 
Echeburúa y sus colaboradores apuntan también a que las consecuencias de los traumatismos pueden ir mas allá de lo habitualmente reconocido (Echeburúa, 2004; Echeburúa, Corral y Amor, 2002).

Sin afán de exhaustividad señalaremos que hay datos que indican la presencia de traumatismos en los trastornos disociativos (Gershuny y Thayer, 1999; Pérez y Galdón, 2003), los trastornos de personalidad, especialmente el limite (Sabo, 1997), la bulimia y otros trastornos alimentarios (Everyll y Waller, 1995; Everyll, Waller y MacDonald, 1995; Terr, 1995), los trastornos de ansiedad (Mancini, Van Ameringen y MacMillan, 1995), los trastornos del estado de animo (Hall, Sachs, Rayens y Lutenbacher, 1993), el alcoholismo (Windle, Windle, Scheidt y Miller, 1995) y muchos otros (véase para una revisión completa el texto de Everly y Lating (1995).

\section{LAS CONTRADICCIONES INTERNAS DEL DSM-IV Y LA CIE-10}

Como es bien sabido los manuales de diagnóstico habitual se declaran «ateóricos» y este principio conceptual implica, ente otras muchas cosas, que no se mencionan los agentes etiológicos de la inmensa mayoría de los trastornos mentales y del comportamiento que en los mismos se detallan. Una revisión mas detallada de ambos manuales nos muestra que, efectivamente, el papel patógeno del estrés y de los traumas se menciona muy escasamente.

Así, el DSM-IV-TR (APA, 2000) solo cita el estrés y el trauma en algunas categorías diagnosticas, a saber: «trastorno por estrés agudo» "trastorno por estrés postraumático», «trastorno psicótico breve», «trastornos adaptativos» $y$ «vaginismo». La CIE 10 (OMS, 1992) propone la especificación «con estrés agudo asociado» en los "trastomos psicóticos agudos y transitorios»; y tiene una categoría general llamada «reacciones a estrés grave y trastornos de adaptación», donde se incluyen ureacción a estrés agudo», el «trastorno por estrés postraumático» y el «trastorno adaptativo». A destacar la categoría «transformación de la personalidad tras una experiencia catastrófica» que se concibe como una consecuencia posible del «trastorno por estrés postraumático». Ambos manuales, no obstante, permiten asociar el estrés con las patologías diagnosticadas mediante la adición de códigos reseñados ya sea en el eje IV, en el caso del sistema DSM, o en el eje III, de la CIE.
Esta adición, sin embargo, es presentada en ambos manuales como un dato concomitante más a tener presente, pero sin excesivo peso en el diagnóstico en cuestión al que se añaden.

Pero a pesar de la supuesta orientación ateórica y «aetiológica», si se nos permite el uso de semejante vocablo, ambos manuales mencionan, tal como deciamos, el estrés, ya sea en su vertiente más estándar o en su aspecto más vinculado a experiencias traumáticas, como factor causal implicado en los trastornos citados. Creemos observar aquí una primera contradicción o fuente de confusión. Resulta que declarándose manuales ateóricos afirman, no obstante, que algunos trastornos están vinculados al estrés y los traumas. Con esta afirmación se esta dando, de modo implícito, la idea de que unos trastornos lo están y otros no. ¿Porque no se cita el estrés como factor patógeno en ciertos tipos de depresiones, por ejemplo? ¿O en otros trastornos de ansiedad? ¿O es que hay hoy día algún profesional de la salud mental que mantenga dudas razonables sobre el papel del estrés en ciertos tipos de depresión o trastornos de ansiedad? Sin afán de exhaustividad mencionar tan solo que existe evidencia tanto a nivel de la patofisiología animal (Fuchs, Czéh, y Flügge, 2004; van Kampen, Kramer, Hiemke, Flügge, y Fuchs, 2002) y humana (Sher, 2004), como a nivel de la clínica psicopatológica (Nutt, 2004; Rojo, Livianos, Cervera, Domínguez y Reig 2002; Tennant, 2001).

Podríamos aportar mas pruebas sobre la falta de consistencia de la (ateoricidad» del sistema DSM. Algunas son especialmente llamativas. Véase, por ejemplo, el «trastorno reactivo de la vinculación de la infancia o la nifiez) (APA, 2000) (equivalente a F94.1 y F94.2 de la CIE 10; OMS, 1992). En la definición y criterios de dicho trastorno se da una clara afirmación sobre la etiología del trastorno cuando el manual dice, la cita es textual, el subrayado nuestro (APA, 2000; p. 130):

\begin{abstract}
Por definición, el trastorno se asocia a una crianza claramente patológica que puede adoptar la forma de desatención persistente de las necesidades emocionales básicas del niño relativas a bienestar, estimulación y afecto; desatención persistente de las necesidades fisicas básicas del niño o cambios repetidos del cuidador primario, lo que evita la formación de vínculos estables Se presume que la crianza patológica es responsable de la relación social alterada.
\end{abstract}

Entiéndase nuestra posición. No estamos criticando al sistema DSM por incluir en algunos tras- 
tornos consideraciones de orden etiológico. Bien al contrario; somos partidarios, y en esto creemos estar en sintonía con la mayoría de los estudiosos de la psicopatología, de una clasificación diagnóstica que incluya información sobre la etiología de los trastornos mentales cuando ello sea posible y cuente con la base empírica suficiente. Lo que estamos apuntando es que tal y como están ahora diseñados los manuales al uso se podría incluir mas información sobre el papel patógeno del estrés en general y de los efectos de los traumatismos en particular.

No nos parece demasiado atrevido señalar que, además, los traumas o el estrés no son conceptos teóricos. Mas bien al contrario; sostenemos que son hechos clínicos, en muchas ocasiones más fáciles de objetivar o tan objetivables, al menos, como muchos de los síntomas (que no signos) que se utilizan para el diagnóstico psicopatológico; la tristeza o la dificultad para conciliar el sueño, por poner solo un par de ejemplos muy comunes. Creemos que en esta cuestión radica una segunda contradicción interna de los manuales de diagnóstico que conviene destacar.

\section{PERFILANDO LOS CONCEPTOS DE ESTRÉS Y TRAUMA}

A pesar de que hace años que se maneja el concepto de estrés, su definición sigue siendo una cuestión compleja. De acuerdo con Sandín (1995), podemos entender el estrés desde una triple perspectiva. La estimular (aquella que pone el énfasis en el agente estresante. La de la respuesta) incidiendo en el esfuerzo adaptativo del sujeto ya sea a nivel psicofisiológico o comportamental. Y la interaccional —poniendo en juego la relación entre estimulo y sujeto, esto es, valorando el factor subjetivo como determinante de la respuesta final.

Sería muy tentador situarnos en la primera perspectiva y efectuar las especificaciones diagnósticas que proponemos teniendo en cuenta exclusivamente los estímulos que recibe el sujeto. Podríamos, entonces, anotar sin más un mero listado de situaciones psicosociales o eventos vitales que conllevan cambios en la vida del individuo. Pero nos parece una aproximación insuficiente. Nos situamos más próximos a la propuesta de Lazarus (Lazarus y Folkman, 1984) de definir el estres como cualquier suceso en el que las demandas ambientales $o$ internas (o ambas) sobrecargan o exceden las posibilidades adaptativas del sujeto y conllevan, pues, la idea de amenaza para el individuo.

Esta definición es operativa en tanto en cuanto tiene en cuenta los estímulos ambientales, perfectamente objetivables, aproximándonos entonces al concepto de «acontecimiento vital» (Holmes y Rahe, 1967); así como los factores individuales de respuesta a los mismos, generando de este modo una perspectiva claramente interaccional entre el entorno y las capacidades de respuesta del sujeto (Magnusson y Öhman, 1987; Zubin y Spring, 1977).

Idéntica posición adoptamos con respecto a la consideración del fenómeno traumático. Echeburúa (2004) distingue entre «suceso traumático" (desde la perspectiva del estímulo propiamente dicho) y «trauma» (desde la respuesta del sujeto). Por «suceso traumático» entiende un acontecimiento negativo e intenso que surge de forma brusca, que resulta inesperado e incontrolable y que, al poner en peligro la integridad fisica o psicológica de una persona que se muestra incapaz de afrontarlo, tiene consecuencias dramáticas para la víctima, especialmente de terror e indefensión. Considera utrauma» la reacción psicológica derivada de un suceso traumático.

Una definición interaccional del trauma implica tanto la consideración del estímulo como la de la respuesta del sujeto. En este sentido, hay un cierto consenso (Davidson y Foa, 1993; Classen, Koopman y Spiegel, 1993; Herman, 1997; March, 1993; Spiegel y Cardena, 1991) en considerar que un trauma se da cuando un individuo se ve envuelto en hechos que representan un peligro real para su vida o cualquier otra amenaza para su integridad fisica; o bien cuando se es testimonio de un acontecimiento donde se producen muertes, heridos, o existe una amenaza para la vida de otras personas. $Y$ a ello se añade la idea de que la respuesta del sujeto a este acontecimiento es de temor, desesperanza y horror intensos ya que el evento o eventos en cuestión superan la capacidad de respuesta de la persona y sus mecanismos habituales de afrontamiento $y$ defensa. Esta definición de trauma, que es la que rocoge el DSM-IV (APA, 2000), nos parece suficientemente operativa para nuestros propositos.

No obstante, y con vistas a la propuesta de especificadores diagnósticos asociados al trauma, nos parece interesante tener presentes ciertos matices que, desde la perspectiva estimular, se pueden efec- 
tuar al concepto de trauma. Terr (1991), por ejemplo, propone discriminar entre trauma «tipo I y II". En el primero se da un evento puntual (como una violación o la visión de un asesinato). En el segundo se sufre una exposición repetida a eventos extremos. Solomon y Heide (1999) añaden un tercer tipo, más severo aun. Definen una situación extrema, repetida y crónica pero que se produce desde temprana edad y con especial sadismo. Citan como ejemplos niños sometidos a constantes abusos fisicos y sexuales, con empleo de fuerza, tortura, amenazas a sus seres queridos, ritos sectarios, etc.

Para estos autores la utilidad de la clasificación radica en que cada tipo de trauma requiere un abordaje diferente puesto que presenta una clínica postraumática diferente. Así en el caso del trauma «tipo I» el paciente se presenta, por lo general, con un recuerdo claro de lo sucedido y las terapias más breves, del tipo que sean, pueden ser de utilidad. Los pacientes del «tipo II» acuden a la consulta con una patología muy variada, de orden depresivo fundamentalmente, con defensas como la negación, la represión y la disociación en pleno funcionamiento; su tratamiento es más complejo, largo y delicado. Los pacientes del «tipo III» pueden recibir diagnósticos como esquizofrenia, trastorno límite de la personalidad, trastorno bipolar o trastorno de identidad disociativo; son típicas también las múltiples somatizaciones (ente las que destaca el dolor de cabeza de origen desconocido) y una notable amnesia disociativa con respecto a importantes sectores de la infancia (Solomon y Heide, 1999).

Para nosotros, como deciamos anteriormente, en el contexto de este trabajo, nos parece de utilidad poder diferenciar entre estos tres tipos de estimulación traumática con vistas a una mayor precisión de los especificadores diagnósticos que a continuación proponemos.

\section{PROPUESTA DE ESPECIFICADORES DIAGNÓSTICOS VINCULADOS AL ESTRÉS Y EL TRAUMA}

Tal y como es sabido las especificaciones permiten definir subgrupos más homogéneos de individuos que sufren un trastomo y que comparten ciertas caracteristicas. Una vez establecido el diagnóstico, pueden aplicarse ciertos especificadores indicadores de gravedad y curso evolutivo, a saber: leve, moderado, grave, en remisión parcial, en remisión total e historia anterior. En el sistema DSM cabe contemplar cómo varios trastornos cuentan con criterios especificos para definirlos de modo mas ajustado: "retraso mental», "trastorno disocial», «fobia social», «trastorno obsesivo compulsivo», «trastorno por estrts postraumatico», "trastomo psicótico breve», "episodio maníaco» y "episodio depresivo mayon, entre otros. Algunos, por ejemplo, cuentan con criterios especificos para definirlos en remisión parcial o en remisión total: "episodio maníaco», «episodio depresivo mayon y "dependencia de sustancias», por ejemplo.

Los especificadores, pues, pueden ser de diferentes tipos: unos son de orden temporal y describen el curso del trastorno, otros registran la gravedad del trastorno y los hay destinados incluso a señalar el nivel de conciencia del trastorno. Nada impediría entonces poder añadir especificadores que tuvieran en cuenta lo dicho hasta aquí con respecto al estrés y los traumas y su relación con la psicopatología. Somos conscientes de que hasta ahora hemos insistido en el papel etiopatogénico de ambos conceptos. La propuesta de empleo de estos especificadores es, no obstante, más humilde y pretende, tan sólo, un reflejo diagnóstico más adecuado de la cuestión. La etiología de los trastornos mentales nos parece un asunto de tal complejidad que

Tabla 1. Propuestas de nuevas especificaciones

Propuesta de especificadones del estres:

Trastorno $x$... vinculado a estrés agudo

Trastorno $x$... vinculado a estrés crónico

Trastorno $x$... vinculado a trauma (especificando tipo de trauma I, II o III)

Propuesta de especificadores de factores asociados al estrés: 
dificilmente puede, por el momento, quedar reflejado en un rotulo diagnóstico como los que usamos hoy en día. Nuestra propuesta consiste en que los manuales de diagnóstico al uso añadan las especificaciones siguientes en la mayoría de los trastornos (véase la Tabla 1).

Explicaremos sucintamente que entendemos por cada uno de estos especificadores. Entendemos por estrés agudo las situaciones de sobrecarga producidas por acontecimientos vitales puntuales de carácter no masivo ni catastrófico pero que pueden despertar en la persona una reacción emocional intensa y/o un esfuerzo de adaptación a una situación nueva. Un ejemplo lo tendriamos en una intervención quirúrgica o un cambio en la situación laboral. Entendemos por estrés crónico las situaciones de sobrecarga producidas por situaciones vitales (nótese la diferencia con «acontecimientos») de la definición anterior) permanentes o de larga duración, de carácter no masivo ni catastrófico, pero que pueden despertar en la persona una reacción emocional intensa y/o un esfuerzo de adaptación de larga duración. Posibles ejemplos serian la inmigración, el desempleo, la enfermedad crónica, la jubilación, el nacimiento de un hijo, etcétera. Por trauma considerariamos aquí la situación que se da cuando una persona se ve envuelta en hechos que representan un peligro real para su vida o cualquier otra amenaza para su integridad fisica; o bien cuando es testimonio de un acontecimiento donde se producen muertes, heridos, o existe una amenaza para la vida de otras personas. La respuesta del sujeto a este acontecimiento es de temor, desesperanza y horror intensos. El subtipo de trauma ha quedado definido unas líneas más arriba.

Proponemos, como se ha visto, utilizar especificadores de carácter temporal que permitan matizar mejor la relación entre el estrés y posibles traumas y la condición psicopatológica del paciente. Utilizaremos la especificación como factor predisponente cuando no sea posible establecer una relación temporal clara y definida entre el agente estresante o traumático y el inicio de las dificultades del paciente. Utilizaremos la especificación como factor precipitante cuando sea posible establecer una relación temporal clara y definida entre el agente estresante o traumático y el inicio de las dificultades del paciente. Por último se podría usar la especificación como factor de mantenimiento_cuando el agente estresante o traumático este presente en la actualidad y el clinico considere que contribuye -aunque no sea el único factor- a la vigencia del trastorno en cuestión, dificultando su resolución.

Consideramos que con estas especificaciones propuestas se podrían plantear diagnósticos mas ajustados a la situación clínica del paciente. No es lo mismo el diagnostico de «trastorno depresivo mayor, episodio único, moderado»; que el de «trastorno depresivo mayor, episodio único, moderado, vinculado a estrés crónico, como factor predisponente», por poner solo un ejemplo. No es únicamente el elemento puramente nosológico el que se beneficiaría de la aplicación de esta propuesta; colocar en un primer plano los elementos que estos especificadores realzan conlleva importantes beneficios terapéuticos.

\section{CONSIDERACIONES FINALES}

Los sistemas DSM y CIE poseen formatos de diagnóstico multiaxial en los que se puede consignar la presencia de agentes estresantes y/o traumáticos para cada caso en particular. Nuestra propuesta de especificadores bien podría tener cabida tanto en el eje IV del DSM como en el eje III del sistema CIE. Sin embargo consideramos que sería preferible que estos especificadores pudieran ser consignados en el eje I de los respectivos manuales. Por diversas razones.

En primer lugar, el empleo multiaxial es opcional para los usuarios de estos manuales. Ello significa que se pueden realizar diagnósticos que no recojan los aspectos comentados. Es decir, pueden emplearse dejando completamente al margen las consideraciones relativas al estrés y los traumas, amén de cualquiera otra de raigambre psicosocial.

En segundo lugar, la disposición de los ejes que permiten vincular estos problemas con las patologías a diagnosticar invita a efectuar una consideración de los mismos de carácter global, tal y como puede observarse en los ejemplos que los propios manuales muestran para ilustrar el empleo de sus respectivos ejes. Esto es, un paciente que recibe más de un diagnóstico en el eje I es evaluado, desde el punto de vista de los problemas psicosociales (estrés y traumas incluidos), de modo unitario. No es posible, entonces, vincular de modo más concreto y especifico los problemas psicosociales detectados con cada uno de los diagnósticos realizado. 
Somos conscientes que establecer un vínculo tan directo resulta imposible en muchas ocasiones. Pero también de que debemos hacer un esfuerzo por intentar determinar con la mayor precisión las relaciones entre el estrés y/o los traumas con el sufrimiento psicopatológico de nuestros pacientes.

$Y$ es que en nuestra opinión, el hecho de obviar el estrés y los traumas como posibles elementos a tener en cuenta en la etiología y el diagnóstico de muchos trastornos mentales no responde a una realidad clínica sino a una realidad de otro orden. Una realidad coyuntural, globalizada, medicalizada y economicista, una realidad que tiende a ver los trastornos mentales como enfermedades de las cuales solo el sujeto es portador; enfermedades en el término más organicista de la palabra, ausentes de contacto con el medio en el que esta el sujeto, su biografia, sus relaciones y los riesgos que asume por el hecho de vivir en sociedad.

No cabe duda de que esta situación esta multideterminada. Los sistemas DSM y CIE contribuyen pero no son el único factor que interviene. Los propios pacientes, por ejemplo, tienen, en muchas ocasiones, tendencia a no mencionar sus acontecimientos vitales o los traumatismos sufridos, bien sea por vergüenza, por culpa, por no rememorar el sufrimiento pasado, por no establecer la conexión ente el agente estresante y su malestar o por falta de confianza (o de tiempo) con su entrevistador.

Estas y otras razones (falta de entrenamiento y formación de clínico, complicación del tratamiento farmacológico e incluso posibles problemas legales) nos parece que son las que explican porque no se reconocen adecuadamente, en muchos casos, los factores estresantes y/o traumáticos en la patología de un buen número de pacientes.

Las consecuencias de este fenómeno que estamos señalando son importantes. Para la ciencia y para los pacientes. El diagnóstico psicopatológico se ve privado, en muchos casos, de un elemento capital. Y los pacientes pueden recibir dosis de medicación excesiva o mal orientada, continúan arrastrando las consecuencias del estrés y los traumas durante más tiempo y no reciben el apoyo terapéutico, ni social, del que serían merecedores.

\section{REFERENCIAS}

American Psychiatric Association (1952). Diagnastic and statistical manual of mental disorders 1 . Washington: APA.
American Psychiatric Association (2000). Diagnostic and statistical manual of mental disonders IV. Text Revision. Washington: APA.

Barret, J.E., Rose, R.M., y Klerman, G.L. (1979). Stress and mental disorder. New York: Raven Press.

Beck, J.C., y van der Kolk, B. (1987). Reports of childhood incest and current behavior of chronically hospitalized psychotic women. American Journal of Psychiatry, 144, 1474-1476.

Buendia, J. (1993). Estrés y psicopatologia. Madrid: Pirámide.

Classen, C., Koopman, C., y Spiegel, D. (1993). Trauma and dissociation. Bulletin of the Menninger Clinic, 57, 179-194.

Davidson, R.T., y Foa, E.B. (Eds.) (1993). Pastmoumatic stress disorders in review: Recent reseanch and future development. Washington, DC: American Psychiatric Press.

DeLonguis, A., Lazarus, R.S., y Folkman, S. (1988). The impact of daily stress on health and mood: Psychological and social resources as mediators. Joumal of Personality and Social Psychology, 54, 486-495.

Echeburia, E. (2004). Superar un trauma. El tratamiento de las victimas de sucesos violentos. Madrid: Pirámide.

Echeburúa, E., De Corral, P., y Amor, P.J. (2002). Evaluación del daño psicológico en las víctimas de delitos violentos. Psicothema, 14, 139-146.

Everill, J.T., y Waller, G. (1995). Reported sexual abuse and eating psychopathology: A review of the evidence for a causal link. International Journal of Eating Disonders, 18, 1-11.

Everill, J.T., Waller, G., y MacDonald, W. (1995). Reported sexual abuse and bulimic symptoms: The mediating role of dissociation. Dissociation, 8, 155-159.

Everly, G.S., y Lating, J.M. (1995). Psychotraumatology. New York: Plenum Press.

Ferenczi, S. (1908). Interpretación y tratamiento psicoanalitico de la impotencia psicasexual. En Obras Completas (Vol. 1). Madrid: Espasa Calpe.

Ferenczi, S. (1932). Reflexiones sobre el traumatismo. En Obras Completas, Tomo IV. Madrid: Espasa Calpe.

Freud, S. (1897). Carta a Fliess del 21.IX. En Obras Completas, Vol. I. Buenos Aires: Amorrortu.

Friedman, M. (1996). PTSD: Diagnosis and treatment for mental health clinicians. Community Mental Health Journal, 32, 173-189.

Fuchs, E., Czéh, B., y Flügge, G. (2004). Examining novel concepts of the pathophysiology of depression in the chronic psychosocial stress paradigm in tree shrews. Behavioural Pharmacology, 15, 315-25.

Gershuny, B.S., y Thayer, J.F. (1999). Relations among psychological trauma, dissociative phenomena, and trauma-related distress: $\mathrm{A}$ review and integration. $\mathrm{Cli}$ nical Psychology Review, 19, 631-657.

Hall, L.A., Sachs, B, Rayens, M.K., y Lutenbacher, M. (1993). Childhood physical and sexual abuse: Their relationship with depressive symptoms in adulthood I mage: The Journal of Nursing Scholarship, 25, 317-323. 
Herman, J. (1997). Trauma and necovery. The aftermath of violence from domestic abuse to political terror. New York: Basic Boks.

Holmes, T.H., y David, E.M. (1989). Life change, life events and illness. New York: Praeger.

Holmes, T.H., y Rahe, R.H. (1967). The social readjustment rating scale. Journal of Psychosomatic Research, $11,213-218$.

Jarne, A. (2000). Trastornos adaptativos. En A. Jarne y A. Talarn (Eds.), Manual de psicopatologia clinica (pp. 681-707). Barcelona: Paidós.

Jaspers, K. (1913). Psicopatologia genenal. Buenos Aires: Beta, 1973. (Edición original en alemán de 1913).

Kardiner (1941). The traumatic neurosis of war. New York: Hoeber.

Kraepelin, E. (1883). Compendium der Psychiatrie. Leipzig: Abel.

Kraepelin, E. (1899). Psychiatrie. Leipzig: Barth.

Lazarus, R.S. (1966). Psychological stress and the coping process. New York: McGraw-Hill.

Lazarus, R.S., y Folkman, S. (1984). Stress, appraisal and coping. New York: Springer.

Magnusson, D., y Ohman, A. (1987). Psychopathology: An interactional perspective. London: Academic Press.

Mancini, C., Van Ameringen, M., y MacMillan, H. (1995). Relation of childhood sexual and physical abuse to anxiety disorders. Journal of Nervous and Mental Disease, 183, 309-314.

March, J.S. (1993). What constitutes a stress? The criterion A issue. En Davidson, J.R.T., y Foa, E.B. (Eds.), Posttraumatic stress disorder: DSM-IV and beyond (pp. 37-54). Washington: American Psychiatric Press.

McFarlane, A.C. (2004). The contribution of epidemiology to the study of traumatic stress. Social Psychiatry and Psychiatric Epidemiology, 39, 874-882.

Meyer, A. (1957). Psychobiology: A science of man. Springfield: C.C. Thomas.

Muenzenmaier, K., Meyer, I., y Struening, E. (1993). Childhood abuse and neglect among women outpatients with chronic mental illness. Hospital and Community Psychiatry, 44, 666-670.

Mueser, K., Salyers, M., y Rosenberg, S. (2001). Psychometric evaluation of trauma and posttraumatic stress disorder assessments in persons with severe mental illness. Psychological Assessment, 13, 1111-1117.

Neufeld, R.W.J. (1982). Psychological stress and psychopathology. New York: McGraw-Hill.

Nutt, D. (2004). Anxiety and depression: individual entities or two sides of the same coin. International Journal of Psychiatry in Clinical Practice, 8, 19-24.

Organización Mundial de la Salud (1992). The ICD-10 Classification of Mental and Behavioural Disorders. Geneve: O.M.S.

Pavlov, I.P. (1927). Conditioned neflexes. London: Cambridge University Press.

Ptrez, S., y Galdón, M.J. (2003). Los fenómenos disociati- vos: una revisión conceptual. Revista de Psicopatologia y Psicologia Clínica, 8, 85-108.

Rojo, L., Livianos, L., Cervera, G., Dominguez, J.A., y Reig, M.J. (2002). The role of stress in the onset of depressive disorders. A controlled study in a Spanish clinical sample. Social Psychiatry and Psychiatric Epidemiology, 37, 592-5.

Sabo, A.N. (1997). Etiological significance of association between chilhood trauma and borderline personality disorder: Conceptual and clinical implications. Journal of Personality Disonders, 11, 50-70.

Sandín, B. (1995). El estrés. En A. Belloch., B. Sandín y F. Ramos (Eds.), Manual de psicopatologia (Vol. 2, pp. 352). Madrid: McGraw-Hill

Sandin, B., y Chorot, P. (1993). Stress and anxiety: Diagnosis validity of anxiety disorders according to life event stress, ways of coping and physical symptoms. Psiquis, 14, 178-184.

Schneider, K. (1946). Patopsicologia clinica. Madrid: Paz Moltalvo, 1975. (Edición original en alemán de 1946).

Selye, H. (1936). A syndrome produced by diverse nocuous agents. Nature, 138, 232-238.

Selye, H. (1956). The stress of life. New York: McGraw-Hill.

Sher, L. (2004). Daily hassles, cortisol, and the pathogenesis of depression. Medical Hypotheses, 62, 198-202.

Solomon, E.P., y Heide, K.M. (1999). Type III trauma: toward a more effective conceptualization of psychological trauma. International Journal of Offender Thenapy and Comparative Criminology, 43, 202-210.

Spiegel, D., y Cardena, E. (1991). Disintegrated experience: The dissociative disorders redefined. Journal of Abnormal Psychology, 100, 366-378.

Tennant, C. (2001). Work-related stress and depressive disorders. Joumal of Psychosomatic Research, S, 697-704.

Terr, L.C. (1991). Childhood traumas: An outline and overview. American Journal of Psychiatry, 148, 10-20.

Terr, R.C. (1995). Childhood traumas: An outline and overview. En G.S. Everly y J.M. Lating (Eds.), Psychotraumatology (pp. 301-320). New York: Plenum Press

Tucker, W.M. (2002). How to include the trauma history in the diagnosis and treatment of psychiatric inpatients. Psychiatric Quaterly, 73, 135-144.

van der Kolk, B.A., McFarlane, A.C., y Weisaeth, L. (1996). Traumatic stress: The effects of overwhelming experience on mind, body and society. New York: Guilford.

van Kampen, M., Kramer, M., Hiemke, C., Flügge, G., y Fuchs, E. (2002). The chronic psychosocial stress paradigm in male tree shrews: evaluation of a novel animal model for depressive disorders. Stress: The International Journal on the Biology of Stress, 5, 37-46.

Windle, M., Windle, R.C., Scheidt, D.M., y Miller, G.B. (1995). Physical and sexual abuse and associated mental disorders among alcoholic inpatients. American Journal of Psychiatry, 152, 1322-1328.

Zubin, J. y Spring, B. (1977). Vulnerability: A new view of schizophrenia. Journal of Abnormal Psychology, 86, 103-126. 